\title{
Diffuse synchrotron emission from galactic cosmic ray electrons
}

\author{
G. Di Bernardo ${ }^{1}$, D. Grasso ${ }^{2}$, C. Evoli ${ }^{3}$, and D. Gaggero ${ }^{4,5}$ \\ ${ }^{1}$ MPI für Astrophysik, Karl-Schwarzschild-Strasse 1, 85740 Garching, Germany \\ ${ }^{2}$ Istituto Nazionale di Fisica Nucleare, Sezione di Pisa, Largo B. Pontecorvo, 56127 Pisa, Italy \\ ${ }^{3}$ II. Institut für Theoretische Physik, Universität Hamburg, Luruper Chaussee 149, 22761 Hamburg, Germany \\ ${ }^{4}$ SISSA, Via Bonomea 265, 34136 Trieste, Italy \\ ${ }^{5}$ INFN, sezione di Trieste, via Valerio 2, 34127 Trieste, Italy \\ Correspondence to: G. Di Bernardo (bernardo@mpa-garching.mpg.de)
}

Received: 17 April 2015 - Revised: 28 July 2015 - Accepted: 9 September 2015 - Published: 22 September 2015

\begin{abstract}
Synchrotron diffuse radiation (SDR) emission is one of the major Galactic components, in the $100 \mathrm{MHz}$ up to $100 \mathrm{GHz}$ frequency range. Its spectrum and sky map provide valuable measure of the galactic cosmic ray electrons (GCRE) in the relevant energy range, as well as of the strength and structure of the Galactic magnetic fields (GMF), both regular and random ones. This emission is an astrophysical sky foreground for the study of the Cosmic Microwave Background (CMB), and the extragalactic microwave measurements, and it needs to be modelled as better as possible. In this regard, in order to get an accurate description of the SDR in the Galaxy, we use - for the first time in this context - 3-dimensional GCRE models obtained by running the DRAGON code. This allows us to account for a realistic spiral arm pattern of the source distribution, demanded to get a self-consistent treatment of all relevant energy losses influencing the final synchrotron spectrum.
\end{abstract}

\section{Introduction}

Deflection of ultra-high energy cosmic rays (UHECR), rotation measure, synchrotron radiation, and polarized dust are just a small sample of different methods of observation of the galactic magnetized interstellar medium (ISM). Cosmic rays (CRs) are, doubtless, a unique probe of the ISM properties. Thanks to a set of successful experiments such as FermiLAT, PAMELA, AMS-02, the last few years have witnessed an incredible progress in the science of electron, and positron Galactic CRs, over a wide range of energy, from $\mathcal{O}(\mathrm{TeV})$ down to tens of MeV. Unfortunately, solar modulation complicates matters, since the CR spectra observed on Earth are - for $E \lesssim 20 \mathrm{GeV}$ - completely reshaped with respect to their local interstellar spectra (LIS).

Relativistic cosmic ray electrons and positrons (CRE), spiralling around the interstellar magnetic field lines, are at the origin of the radio diffuse emission from the Milky Way. For magnetic field intensity of $\mathcal{O}(\mu \mathrm{G})$, like in the case of our Galaxy, and for electrons/positrons of [ $\mathrm{GeV}-\mathrm{TeV}]$ energies, the synchrotron emission falls in the $[\mathrm{MHz}-\mathrm{GHz}]$ range. In- deed, the SDR offers valuable complementary checks of the low energy spectrum, and in general of the spatial distribution of CRs in the Galaxy. Hence, a parallel study of radio emission, together with $\mathrm{CR}$ measurements, can put better constraints on all the interstellar medium (ISM) components involved (Strong et al., 2011). The interpretation of those measurements requires a proper modelling of injection, propagation and losses in the Galaxy.

Moreover, the presence in the [20-200] GHz range of several astrophysical sky signal components - with similar intensities and some spatial correlation - makes the extraction of the CMB a complex task. In order to achieve sufficient accuracy on the cosmological signal the component separation needs to take advantage of the knowledge on the properties of diffuse Galactic emission.

We plan to accomplish the aforementioned study by running the DRAGON code in its 3-dimensional version. Indeed, this is well suited to model the CRE propagation, when accounting for a realistic spiral arm distribution of astrophysical sources, gas distributions, magnetic fields models and dif- 
ferent position-dependent models for diffusion in the parallel and perpendicular directions with respect to the GMF.

\section{Objectives and method}

In the present Section, we outline the guidelines of the multiwavelength analysis we have performed, in order to model the CRE spectra consistently with the diffuse synchrotron emission of the Galaxy. One of our main aims has been:

1. To explore the physical properties - injection and propagation - of the local interstellar spectrum (LIS) of CRE, in the realm of low energies $(\lesssim 7 \mathrm{GeV})$, by combining the latest $e^{-}$and $e^{+}$measurements with the diffuse Galaxy radio emission, between $10 \mathrm{MHz}$ and few $\mathrm{GHz}$. Below that energy, we modelled the LIS of $e^{+}+e^{-}$on the basis of the observed synchrotron spectrum of the Galaxy, which is unaffected by propagation in the heliosphere (see also e.g. Orlando and Strong, 2013).

2. In parallel to that, the current study has pushed us to give an important constrain on the vertical scale height of the diffusion region in the Galaxy, by looking simultaneously at the radio spectrum, the latitude profile of the synchrotron emission, and the positron fraction at energies below $\sim 5 \mathrm{GeV}$.

The structure of the GMF is still not well understood. Generally, a realistic, and accurate description of the synchrotron emission, as well as of its angular distribution, requires to consider two main components for the GMF: the regular and turbulent ones. Regarding the ordered one, here we rely on a recent model, based on a wide and updated compilation of Faraday rotation measurements (Pshirkov et al., 2011). It consists of two different components: a disc field, with a magnitude in the vicinity of the solar system, $B_{0}$, taken to be $2 \mu \mathrm{G}$, and a toroidal halo field, with a thickness of $\sim[0.2-$ $0.4] \mathrm{kpc}$, and extended - above, and below - out of the galactic plane (GP) for [1-2] kpc. ${ }^{1}$ A new, and much-improved model for the regular GMF has been recently brought to the attention of the community (Jansson and Farrar, 2012). In addition to a disc field and an extended halo field, the peculiarity of this GMF model is its X-shape in the $r-z$ directions. The main implications based on such a ordered GMF model will be addressed in our future work.

Regarding the random component - actually, the main responsible for the diffusion of charged particles in the ISM there is still a poor knowledge about its geometrical structure. As in Han et al. (2004), we have assumed it to fill a thick disk, modelled with an exponential vertical profile, and an effective scale-height $z_{\mathrm{h}}$, accordingly to the equation:

\footnotetext{
${ }^{1}$ We have included the regular field in order to make our model compatible with the current information. However, in our analysis, we have checked that only the halo component $\left(B_{\text {halo }}=4 \mu \mathrm{G}\right)$ for the regular GMF plays, albeit marginal, a role.
}

Table 1. The CRE models considered in the present analysis. The reported values of $\gamma_{\text {inj }}\left(e^{-}\right)$refer to energies below/above $4 \mathrm{GeV}$.

\begin{tabular}{llcrc}
\hline Model & $\delta$ & $v_{\mathrm{A}}\left(\mathrm{km} \mathrm{s}^{-1}\right)$ & $\eta$ & $\gamma_{\text {inj }}\left(e^{-}\right)$ \\
\hline KRA & 0.5 & 15 & -0.4 & $1.6 / 2.5$ \\
\hline KOL & 0.33 & 35 & 1.0 & $1.6 / 2.5$ \\
\hline
\end{tabular}

$B_{\text {ran }}(z)=B_{\text {ran }}(0) \exp \left(-z / z_{\mathrm{h}}\right)$.

For the aforementioned purposes, we first run DRAGON, a new numerical package aiming to solve the diffusion equation of CRs in the Galaxy environment (Evoli et al., 2008; Gaggero et al., 2013).

Differently form other semi-analytical and numerical codes, in this contribution we account for a possible spatial dependence of the diffusion coefficient,

$D(\rho, R, z)=D_{0} \beta^{\eta} f(z)\left(\frac{\rho}{\rho_{0}}\right)^{\delta}$,

$\rho$ being the rigidity of the particle, $\beta$ the particle speed in units of speed of light $c$, and $f(z)$ indicates the spatial dependence of the diffusion coefficient. As predicted by the quasi-linear theory (QLT), that should be related to the fluctuating magnetic field, and hence as $D(z)^{-1} \propto B_{\text {ran }}(z) \propto \exp \left(-z / z_{\mathrm{h}}\right)$.

In this paper, for simplicity, only two representative classes of propagation regimes have been taken in consideration: the KRA (Kraichnan), and the KOL (Kolmogorov). The main parameter characterizing those two models are reported in Table 1.

For each of them, we varied the scale-height of the diffusive halo in the range $z_{\mathrm{h}}=[1-16] \mathrm{kpc}$, and the main diffusive parameters were determined in order to minimize the combined $\chi^{2}$ against the boron-to-carbon ratio and the proton observed spectra.

Finally, at high energies ( $\gtrsim 7 \mathrm{GeV})$, unlike our previous results presented in Di Bernardo et al. (2013), here we fix the spectral index, and the normalization of the injection spectrum of the primary electrons and of the extra-component by tuning our models against the new data, as recently released by PAMELA and AMS-02 collaborations, respectively, rather than on $e^{-}+e^{+}$spectrum measured by FermiLAT (Gaggero et al., 2014). With reference to Fig. 1, we want to make it clear that the assumption of a simple powerlaw (PL) distribution for energetic electrons and positrons, whose sources are smoothly distributed in the entire Galactic disc, leads to large overestimation of their energy densities in comparison with the values deduced when a 3-dimensional spiral arm distributions are used. In our opinion, the impact of this more realistic modelling of the particle distribution on the final synchrotron spectral maps is a crucial issue (see also the comparison between the Figs. 5 and 6). 


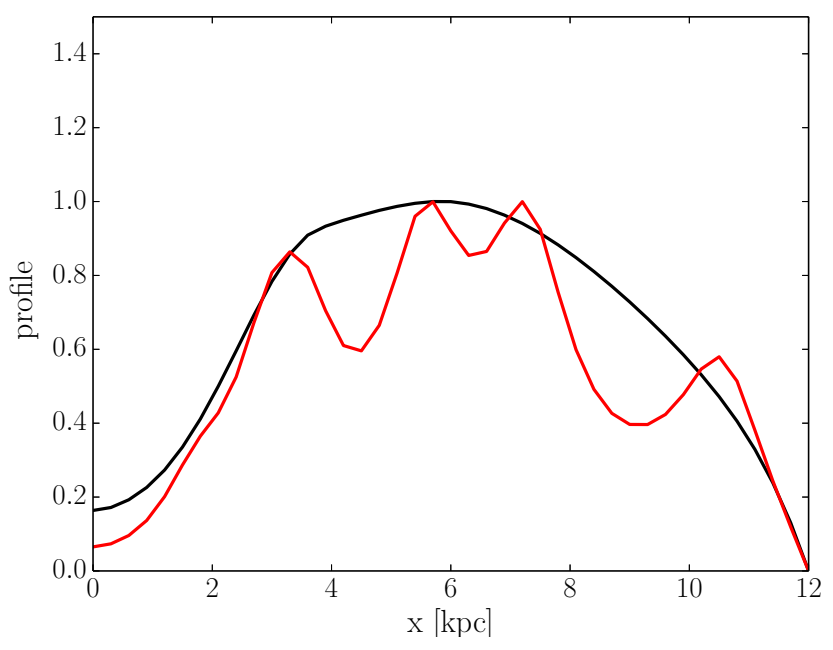

Figure 1. Normalized electron density profile along the radial direction. The black line corresponds to a 2-dimensional, smooth CR. The red one corresponds to a 3-dimensional spiral arm pattern of sources.

\section{The synchrotron emission of the galaxy}

It is known that the synchrotron intensity depends on the spatial, and energetic distribution of CRE density, $n_{\mathrm{e}}$, and the strength of the magnetic field $\left(B_{\perp}\right)$, perpendicular to the line of sight (LOS) to the observer. Once the CRE densities are computed by DRAGON - at all points of the computational grid - we use GAMMASKY, a dedicated code recently used in, e.g. Evoli et al. (2012), and Di Bernardo et al. (2013) to get the emissivities (i.e. power per unit volume per unit frequency per unit solid angle), for the regular and random fields, according to the standard formalism (Longair, 2011), after having integrated over the particle energy.

The emissivity (in $\mathrm{erg} \mathrm{s}^{-1} \mathrm{~Hz}^{-1}$ ) - in an uniform magnetic field - is partially linearly polarized, ${ }^{2}$ and usually subdivided in two components, $\epsilon_{\|, \perp}$, respectively parallel and perpendicular to $B_{\perp} \equiv B(\boldsymbol{r}) \sin \alpha$, with $\alpha$ the angle between the direction of the magnetic field and the LOS. The polarization formulation will be used in our future work. Here, we show results based only on the total intensity, given by

$\epsilon(v, \boldsymbol{r})=\sqrt{3} \frac{e^{3}}{m c^{2}} B_{\perp}(\boldsymbol{r}) F(x) ;$

where $x=v / \nu_{\mathrm{c}}^{\text {reg }}$, being $\nu_{\mathrm{c}}^{\mathrm{reg}}=(3 / 4 \pi)(e / m c) B_{\perp} \gamma^{2}$ the critical synchrotron frequency, $\gamma$ the particle (electron or positron) Lorentz factor, and $F(x)$ is defined in terms of Bessel functions. In the case of a randomly oriented magnetic field, the expected isotropic emissivity is computed according to Ghisellini et al. (1988).

\footnotetext{
${ }^{2}$ For a monochromatic and isotropic distribution of CRE.
}

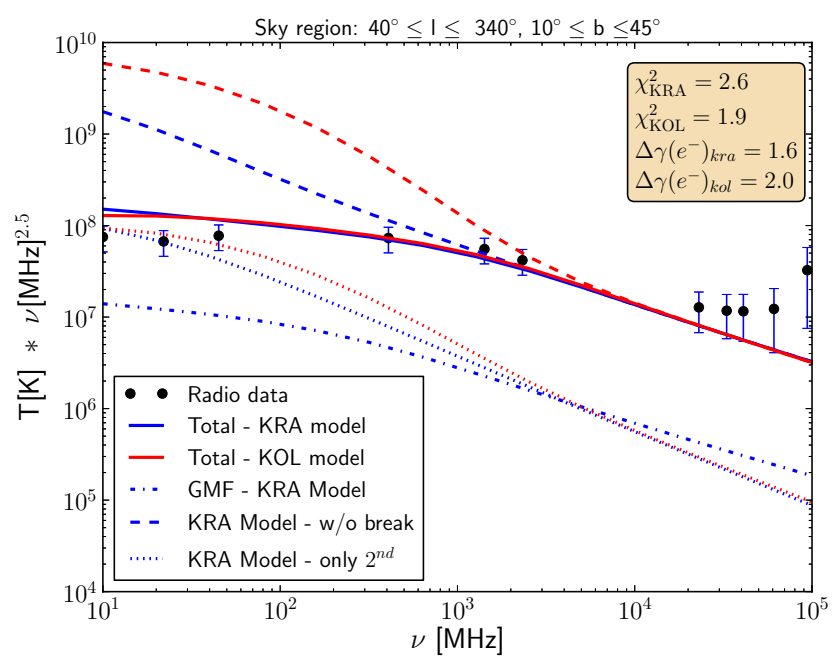

Figure 2. The average synchrotron spectra, for $z_{\mathrm{h}}=4 \mathrm{kpc}$. We show the spectra obtained with (solid lines) and without (dashed lines) the spectral break in the $e^{-}$source spectra. Dotted lines are the contribution of secondary $e^{-}$source spectra. The contribution of the regular GMF is shown as the dot-dashed line. The normalization required for the random component field strength is $B_{\mathrm{ran}}(0)=7.6 \mu \mathrm{G}$.

\subsection{The total synchrotron intensity}

Given a GMF model, and for the representative CRE density models aforementioned, the next step is to compute the Galactic synchrotron spectrum. We take care of correctly reproducing the observed $408 \mathrm{MHz}$ radio synchrotron radiation as in Haslam et al. (1982), by tuning - time to time - the normalization value for the turbulent component of the GMF (see the Eq. 1). We get the sky maps in GAMMASKY by integrating the Galactic emissivity along the LOS,

$I(v)=\int_{\text {1.o.s }} \epsilon(v, \boldsymbol{r}) \mathrm{d} s$,

where $\epsilon(\nu, r)$ is the total emissivity given by Eq. (3). In Fig. 2 we refer to the observed brightness temperature (in K), defined as (Rybicki and Lightman, 1986)

$T(v)=\frac{c^{2} I(v)}{2 k_{\mathrm{B}} v^{2}}$.

The sky maps are subdivided into equal area pixels following the HEALPix ${ }^{3}$ pixelization scheme of Górski et al. (2005). We average the flux over the sky regions $40^{\circ}<l<340^{\circ}, 10^{\circ}<b<45^{\circ},-45^{\circ}<b<-10^{\circ}$, where $l$ and $b$ are Galactic longitude and latitude, respectively. We restrict the analysis to the regions out of the Galactic plane - but avoiding the polar regions - being the contamination from point-like and local extended sources expected to be the smallest. In addition to that, we avoid absorption effect at

\footnotetext{
${ }^{3}$ http://healpix.jpl.nasa.gov/
} 


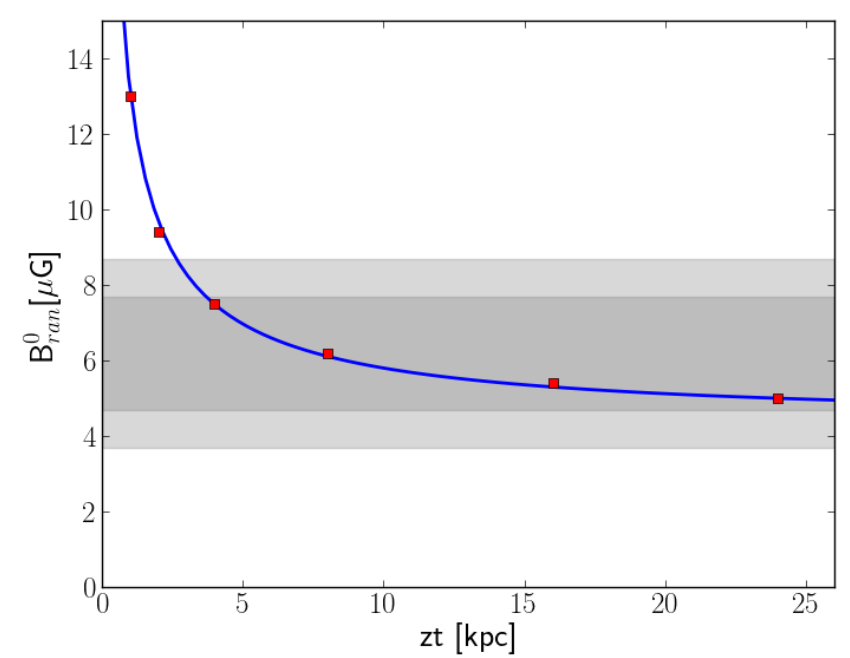

Figure 3. Normalization of random GMF vs. the vertical scale height. The 3(5) $\sigma$ allowed by RM are represented in grey (light grey) bands. The red squares are the values used in our KRA models in order to reproduce the observed spectrum at $408 \mathrm{MHz}$.

radio frequencies, and free-free emission at higher frequencies (Rybicki and Lightman, 1986). Therefore, the observed Galactic diffuse emission in the radio band is, almost entirely, due to the synchrotron radiation of CRE moving errantly in the GMF.

From tens of $\mathrm{MHz}$ to $23 \mathrm{GHz}$ (and up to $94 \mathrm{GHz}$ for WMAP), in such sky region we directly compare our simulated models with the synchrotron spectra measured by a wide set of radio surveys at $22,45,408,1420,2326 \mathrm{MHz}$ as well as WMAP satellite data at 23, 33, 41, 61 and $94 \mathrm{GHz}$.

With reference to Fig. 2, it is immediate to realize that the radio data $(\lesssim \mathcal{O}(100) \mathrm{MHz})$ are clearly incompatible with a single PL electron spectrum, suitable to fit the CRE data. Rather, we find that introducing - below a few $\mathrm{GeV}$ - either a break or an exponential infrared (IR) cut-off in the population of primary $e^{-}$, that helps us in providing a very good description of the radio data.

As immediate consequence of that, the total electron flux (at $E \lesssim 4 \mathrm{GeV}$ ), and hence the radio spectrum below $100 \mathrm{MHz}$, are dominated by secondary particles, which are produced in nuclear collision with the nuclei of the gas present in the ISM, offering thus a direct probe of the interstellar proton spectrum. In this regard, we found that once the low energy $e^{-}$source spectrum is tuned to reproduce the observed $e^{+}$spectrum, only models featuring low reacceleration can reproduce the observed $e^{+}$spectrum and fraction, in total agreement with what found in Strong et al. (2011); Orlando and Strong (2013).

\subsection{The magnetic halo height}

The vertical - perpendicular to the Galactic plane - size of the CR diffusion region represents, undoubtedly, a corner-

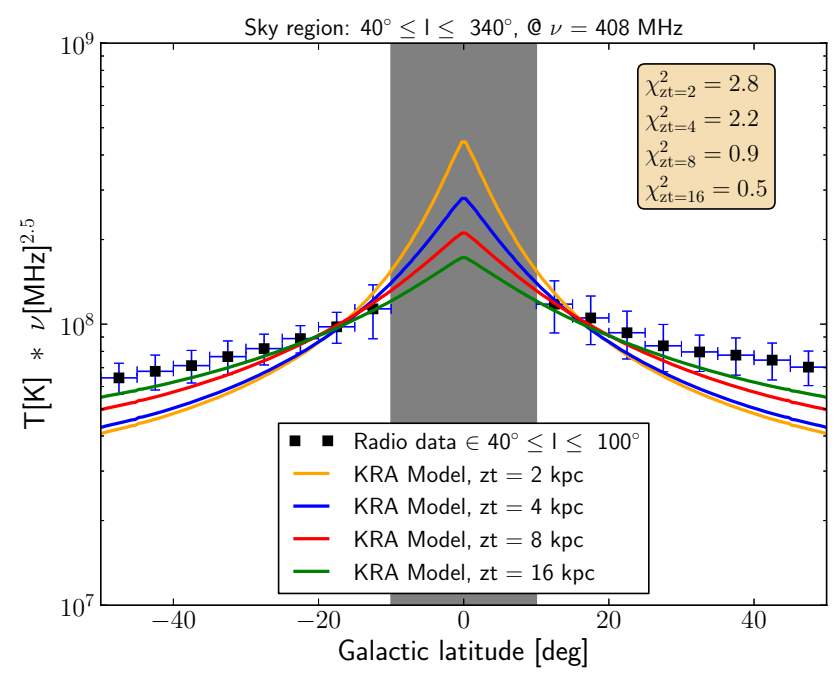

Figure 4. The latitude profile for the synchrotron emission at $408 \mathrm{MHz}$, at different magnetic halo height.

stone in modern Astro-particle physics. The accurate knowledge of it goes beyond the target of conventional CR astrophysics; it is also worth for Dark Matter (DM) indirect search, since that the local flux of DM decay, and annihilation products are expected to depend significantly on such physical length.

So far, the diffusive vertical boundary has been constrained purely on the basis of $\mathrm{CR}$ radioactive nuclide ${ }^{10} \mathrm{Be}$, hence the ${ }^{10} \mathrm{Be} /{ }^{9} \mathrm{Be}$ ratio most commonly. However, this method is subject to the severe uncertainties connected to local distribution of sources, gas, and especially by the solar modulation.

To the contrary, the synchrotron emissivity of the Galaxy offers a much more genuine probe of the scale height $z_{\mathrm{h}}$. In that regards, we may notice that, when a realistic vertical distribution is adopted for the radiation interstellar field (ISRF) and for the GMF, energy losses in the $\mathcal{O}(\mathrm{GeV})$ energy range - hence in the radio energy band - do not affect significantly the CRE vertical distribution, determined predominantly by the diffusion and therefore coincident with that of CR nuclei (see Fig. 5 in Di Bernardo et al., 2013).

Our first argument aiming to constrain the value of $z_{\mathrm{h}}$ is the following one: for a given propagation set up, the synchrotron flux depends, from Eq. (4), only on the random field normalization $B_{\mathrm{ran}}(z=0)$, dominant respect to the regular one, and on $z_{\mathrm{h}}$, the scale-height of the diffusion region $\left(I(v, \boldsymbol{r}) \propto \int B(\boldsymbol{r}) n_{\mathrm{e}}(\boldsymbol{r}) \mathrm{d} s\right)$. As it is possible to appreciate in Fig. 3, the fit of radio data suggests a tight relation $B_{\text {ran }}^{2}(z=0) \propto z_{\mathrm{h}}^{-1}$.

Secondly, we compare the observed latitude profile of the synchrotron emission at $408 \mathrm{MHz}$ to that calculated for the KRA set up, setting different values for $z_{\mathrm{h}}$. For each $z_{\mathrm{h}}$ we tune the value of $B_{\text {ran }}(z=0)$ so that the average spectrum in these regions is reproduced (Fig. 4). Low values of $z_{\mathrm{h}}$ are dis- 


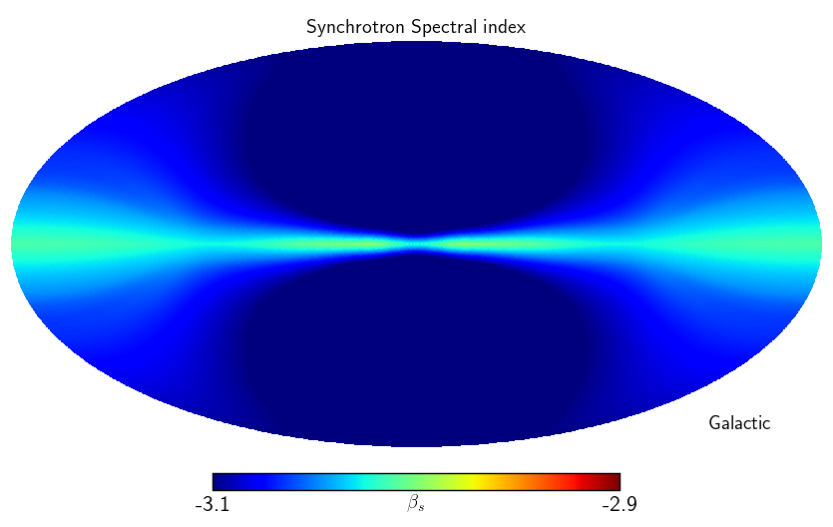

Figure 5. Spectral index map between $408 \mathrm{MHz}$ and $23 \mathrm{GHz}$, with the assumption of a smooth 2-D CR sources. Given the total intensity $I(v) \propto\left(v / v_{0}\right)^{\beta_{\mathrm{s}}}$, the map has been computed according the standard formalism: $\beta_{\mathrm{S}}=0.248 \log \left(I_{23} / I_{408}\right)$.

favoured: a $\chi^{2}$ analysis showed that $z_{\mathrm{h}} \leq 2 \mathrm{kpc}$ are excluded at $3 \sigma$ level.

\section{Future directions and conclusions}

To fully observe and understand the GMF, more effort is required. With our analysis, we exploited the SDR as a way to measure the low energy LIS spectrum of CRE. For the first time, we have placed a constraint on the CR diffusive halo scale height, based on the comparison of the computed synchrotron emission intensity with radio observations. Moreover, we stress out that - for the first time in this framework - our modelling of the SDR emission accounts for the presence of the $e^{ \pm}$charge-symmetric extra-component, required not only to consistently model PAMELA and AMS-02 high energy data, but also to correctly estimate the $e^{-}$source spectrum from CRs and radio data. In our opinion, the combination of high precision CRE data, and current radio observations, can be a viable method to disentangle the contribution of the extra-component to the total synchrotron spectrum.

One of the greatest challenges of observing the Cosmic Microwave Background (CMB) in the [20-200] GHz range resides in the separation between the $\mathrm{CMB}$ and the superimposed foreground emission: free-free, synchrotron, thermal dust. We pointed out that transport of charged relativistic particles, and magnetic fields models should be studied simultaneously, because both have influence on the synchrotron modelling. Synchrotron spectra may reveal signatures of spatially inhomogeneous particle source distributions and magnetic fields. In our treatment here, instead of a smooth CR distribution invariant for rotations about the Galactic disc axis, we rather calculate self-consistently the synchrotron maps emitted by electrons whose spectral density is inhomogeneous, due to all the relevant energy losses sustained while traversing regions with different distributed gas, magnetic and radiation fields.

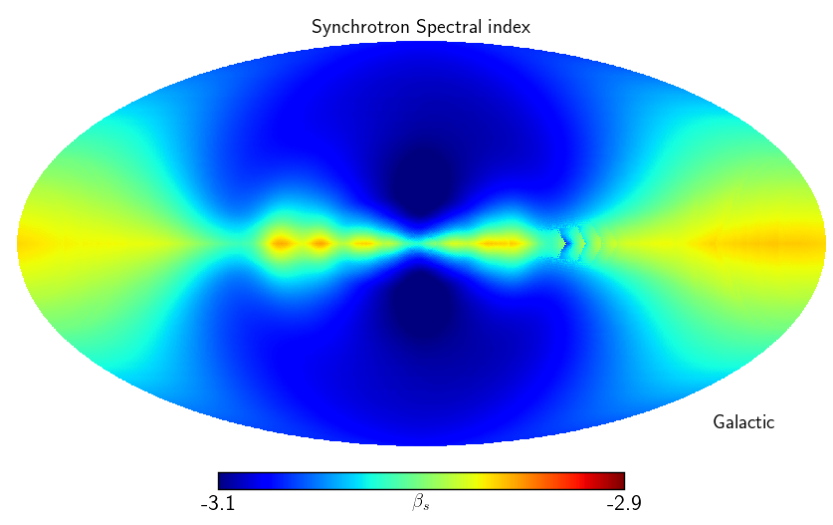

Figure 6. As in Fig. 5, but with the important assumption of a 3-D spiral arm structure.

Finally, the frequency range of GAMMASKY synchrotron simulations, from $\mathcal{O}(10) \mathrm{MHz}$ to $\mathcal{O}(100) \mathrm{GHz}$, covers radio telescopes such as Planck, LOFAR, and SKA. We have developed GAMMASKY code with the aim to support the scientific exploitation of the data provided by these experiments.

Acknowledgements. G. Di Bernardo and C. Evoli would like to thank Klaus Scherer, Julia Becker Tjus and Paolo Desiati for the invitation to the workshop: "Cosmic Ray Anisotropies 2015".

The articleprocessing charges for this open-access publication were covered by the Max Planck Society.

Edited by: P. Desiati

Reviewed by: two anonymous referees

\section{References}

Di Bernardo, G., Evoli, C., Gaggero, D., Grasso, D., and Maccione, L.: Cosmic ray electrons, positrons and the synchrotron emission of the Galaxy: consistent analysis and implications, J. Cosmol. Astropart. Phys., 3, 036, doi:10.1088/1475-7516/2013/03/036, 2013.

Evoli, C., Gaggero, D., Grasso, D., and Maccione, L.: Cosmic ray nuclei, antiprotons and gamma rays in the galaxy: a new diffusion model, J. Cosmol. Astropart. Phys., 10, 018, doi:10.1088/14757516/2008/10/018, 2008.

Evoli, C., Gaggero, D., Grasso, D., and Maccione, L.: Common Solution to the Cosmic Ray Anisotropy and Gradient Problems, Phys. Rev. Lett., 108, 211102, doi:10.1103/PhysRevLett.108.211102, 2012.

Gaggero, D., Maccione, L., Di Bernardo, G., Evoli, C., and Grasso, D.: Three-Dimensional Model of Cosmic-Ray Lepton Propagation Reproduces Data from the Alpha Magnetic Spectrometer on the International Space Station, Phys. Rev. Lett., 111, 021102, doi:10.1103/PhysRevLett.111.021102, 2013.

Gaggero, D., Maccione, L., Grasso, D., Di Bernardo, G., and Evoli, C.: PAMELA and AMS-02 $e^{+}$and $e^{-}$spectra are reproduced 
by three-dimensional cosmic-ray modeling, Phys. Rev. D, 89, 083007, doi:10.1103/PhysRevD.89.083007, 2014.

Ghisellini, G., Guilbert, P. W., and Svensson, R.: The synchrotron boiler, Astrophys. J. Lett., 334, L5-L8, doi:10.1086/185300, 1988.

Górski, K. M., Hivon, E., Banday, A. J., Wandelt, B. D., Hansen, F. K., Reinecke, M., and Bartelmann, M.: HEALPix: A Framework for High-Resolution Discretization and Fast Analysis of Data Distributed on the Sphere, Astrophys. J., 622, 759-771, doi:10.1086/427976, 2005.

Han, J. L., Ferriere, K., and Manchester, R. N.: The Spatial Energy Spectrum of Magnetic Fields in Our Galaxy, Astrophys. J., 610, 820-826, doi:10.1086/421760, 2004.

Haslam, C. G. T., Salter, C. J., Stoffel, H., and Wilson, W. E.: A $408 \mathrm{MHz}$ all-sky continuum survey, II - The atlas of contour maps, Astron. Astrophys., 47, 1, 1982.

Jansson, R. and Farrar, G. R.: A New Model of the Galactic Magnetic Field, Astrophys. J., 757, 14, doi:10.1088/0004637X/757/1/14, 2012.
Longair, M. S.: High Energy Astrophysics, Cambridge University Press, Cambridge, UK, 2011.

Orlando, E. and Strong, A.: Galactic synchrotron emission with cosmic ray propagation models, Mon. Notices Roy. Astron. Soc., 436, 2127-2142, doi:10.1093/mnras/stt1718, 2013.

Pshirkov, M. S., Tinyakov, P. G., Kronberg, P. P., and NewtonMcGee, K. J.: Deriving the Global Structure of the Galactic Magnetic Field from Faraday Rotation Measures of Extragalactic Sources, Astrophys. J., 738, 192, doi:10.1088/0004637X/738/2/192, 2011.

Rybicki, G. B. and Lightman, A. P.: Radiative Processes in Astrophysics, Wiley-VCH, 400 pp., June 1986.

Strong, A. W., Orlando, E., and Jaffe, T. R.: The interstellar cosmicray electron spectrum from synchrotron radiation and direct measurements, Astron. Astrophys., 534, A54, doi:10.1051/00046361/201116828, 2011. 